\title{
LANGUAGES AND THEIR TWO HALVES
}

\author{
Thinh D. Nguyen \\ Email: kosmofarmer@yandex.com
}

\begin{abstract}
We show that the hardness of a language and its two halves can be drastically different.
\end{abstract}

\section{Problem Statement}

Let $\mathbf{L} \subseteq \boldsymbol{\Sigma}^{*}$,

$$
\begin{aligned}
& \frac{1}{2} \mathbf{L}=\left\{\boldsymbol{w} \in \Sigma^{*}\left|\exists v \in \Sigma^{*} \boldsymbol{w} \boldsymbol{v} \in \mathbf{L} \wedge\right| \boldsymbol{w}|=| \boldsymbol{v} \mid\right\} \\
& \mathbf{L} \frac{1}{2}=\left\{\boldsymbol{w} \in \Sigma^{*}\left|\exists \boldsymbol{v} \in \Sigma^{*} \boldsymbol{v} \boldsymbol{w} \in \mathbf{L} \wedge\right| \boldsymbol{w}|=| \boldsymbol{v} \mid\right\}
\end{aligned}
$$

We shall prove that (1) there exists languages $\mathbf{L}$ such that $\mathbf{L} \in \mathbf{P}$, but $\frac{1}{2} \mathbf{L}, \mathbf{L} \frac{1}{2}$ are $\mathbf{N P}$-complete, (2) there exists languages $\mathbf{L}$ such that $\mathbf{L}$ is $\mathbf{N P}$-complete, but $\frac{1}{2} \mathbf{L}, \mathbf{L} \frac{1}{2}$ are in $\mathbf{P}$.

\section{THE LANGUAGE OF PUZZLES AND THEIR ANS WER KEY}

This problem reminds us of the fact that NP problems are "hard to solve but easy to verify". When you have a solution to any NP problem, you can check it in polynomial time.

So, pick an NP-complete language such as HAMPATH i.e. "The set of all directed graphs that have Hamiltonian paths". Define $\mathbf{L}$ to be the language of all strings where the first half is a problem in HAMPATH and the second half is a solution to the problem. We then add filler symbols to make sure the two halves have the same length.

Now, $\mathbf{L}$ is in $\mathbf{P}$ because the solution to an NP-complete problem can be checked in polynomial time. But $\frac{1}{2} \mathbf{L}$ is NP-complete because HAMPATH is an NP-complete language.

\section{THE LANGUAGE OF PUZZLE-PAIRS THAT CONTAIN EACH OTHER'S SOLUTIONS}

Making sure that both halves of the language are NP-complete is a little trickier, but doable. The idea is to make a language of strings $\mathbf{P}_{1} \boldsymbol{x} \mathbf{P}_{2} \boldsymbol{y}$ where $\mathbf{P}_{1}$ and $\mathbf{P}_{2}$ are NP-hard problems, $\boldsymbol{x}$ and $\boldsymbol{y}$ together give a solution key for $\mathbf{P}_{1}$ and $\mathbf{P}_{2}$, but when separated $\boldsymbol{x}$ and $\boldsymbol{y}$ give no useful information. Then you can quickly confirm $\mathbf{P}_{1} \boldsymbol{x} \mathbf{P}_{2} \boldsymbol{y}$ (the language is in $\mathbf{P}$ ), but $\mathbf{P}_{1} \boldsymbol{x}$ and $\mathbf{P}_{2} \boldsymbol{y}$ are just hard problems (both in NP-complete).

Let $\mathbf{L}$ be the language of all strings with the following properties: The first half is a graph $\boldsymbol{G}$ in HAMPATH and an isomorphism $\boldsymbol{G} \rightarrow \boldsymbol{H}$. The second half is a graph $\boldsymbol{H}$ in HAMPATH and a Hamiltonian path in $\boldsymbol{G}$.

If you know $\boldsymbol{G}, \boldsymbol{H}$, an isomorphism $\boldsymbol{G} \rightarrow \boldsymbol{H}$, and a Hamiltonian path in $\boldsymbol{G}$, you can easily confirm whether $\boldsymbol{G}$ and $\boldsymbol{H}$ are Hamiltonian in polynomial time. Hence $\mathbf{L}$ is in $\mathbf{P}$.

If you only know the first half of a string in $\mathbf{L}$, however, you only have a graph $\boldsymbol{G}$ and an isomorphism with another (unknown) graph. Deciding whether $\boldsymbol{G}$ is in HAMPATH is an NP-complete

problem, hence $\frac{1}{2} \mathbf{L}$ is $\mathbf{N P}$-complete. If you only know the second half of a string in $\mathbf{L}$, then you have a 
graph $\boldsymbol{H}$ and a Hamiltonian path for a different (unknown) graph $\boldsymbol{G}$. Without knowing the isomorphism $\boldsymbol{G} \rightarrow \boldsymbol{H}$, we can argue that knowing this Hamiltonian path does not help decide whether $\boldsymbol{H}$ is in Hamiltonian. Hence $\mathbf{L} \frac{1}{2}$ is $\mathbf{N P}$-complete.

\section{THE LANGUAGE OF SIMPLE STRINGS COMBINED WITH A COMPLICATED RULE}

To make both halves easy (in $\mathbf{P}$ ) but the combined string hard (NP-complete), the idea is to find a language consisting of pairs of objects, where each object is separately not too special (one can decide whether it's well-formed in polynomial time), but the rule for pairing them up is complicated and deciding whether the pairing is valid is NP-complete. Hence the language of whole strings will be NP-complete even if the halves are both in $\mathbf{P}$.

The SUB GRAPH-ISO problem is: Given two graphs $\boldsymbol{G}$ and $\boldsymbol{H}$, decide whether $\boldsymbol{G}$ is isomorphic to a subgraph of $\boldsymbol{H}$. This problem is NP-complete. Therefore, let $\mathbf{L}$ be the set of strings which first half is a graph $\boldsymbol{G}$ and the second half is a graph $\boldsymbol{H}$ containing a subgraph isomorphic to $\boldsymbol{G}$. We pad the strings as necessary so that both halves have the same length.

Then $\mathbf{L}$ is NP-complete, but the first and second halves of $\mathbf{L}$ are just the set of all strings corresponding to valid graphs. That's decidable in $\mathbf{P}$.

\section{CONCLUSION}

Garey and Johnson [1] shape their theory based on previous primal works of Cook, Levin and Karp. Johnson [2] moves on with the guide to this theory. As long as we study a mathematical conjecture, we should encourage ourselves to have moderate amount of labour hours on popular mathematics books like these. Then, reading some articles on theory of computing like [3] is a good practice. Having all those done, we could think of the ultimate final for all mathematics sciences.

\section{REFERENCE}

1. Michael R. Garey, David S. Johnson, Computers and Intractability: A Guide to the Theory of NP-Completeness

2. David S. Johnson, The NP-Comple teness Column: An Ongoing Guide

3. Phan Dinh Dieu, Le Cong Thanh, Le Tuan Hoa, Average Polynomial Time Complexity of Some NP-Complete Problems, Theor. Comput. Sci. 46(3): 219-237 (1986) 